\title{
Avaliação do uso de frações indigestíveis do alimento como indicadores internos de digestibilidade em ovinos
}

\section{Gilberto Vilmar Kozloski ${ }^{1}$, Francisco Rondon Mesquita ${ }^{2}$, Tiago Pansard Alves ${ }^{2}$, Douglas de Souza Castagnino ${ }^{2}$, Cristiano Miguel Stefanello², Luis Maria Bonnecarrère Sanchez ${ }^{1}$}

\footnotetext{
${ }^{1}$ Departamento de Zootecnia - Universidade Federal de Santa Maria (UFSM)
}

2 Graduação - Bolsista de Iniciação Científica - UFSM.

RESUMO - Avaliou-se o uso de matéria seca indigestível (MSi) e de fibra detergente neutro indigestível (FDNi) como indicadores internos de digestibilidade em ovinos. Utilizaram-se dados e amostras provenientes de seis ensaios independentes de digestibilidade com ovinos mantidos em gaiolas de metabolismo recebendo à vontade diversos tipos de volumoso e/ou concentrado. Os resíduos indigestíveis (MSi e FDNi) foram determinados após 144 horas de incubação in situ de amostras de alimentos e fezes. O grau de recuperação da MSi variou de 64,8 a 108,5\% e o da FDNi, de 49,5 a 67,9\%. Quando a relação entre a concentração dos indicadores nas fezes e nos alimentos não foi corrigida para a recuperação fecal, a maior parte das estimativas médias de digestibilidade da matéria orgânica dos experimentos foi inferior às médias obtidas in vivo. Quando a relação foi corrigida para a recuperação fecal, as estimativas médias de digestibilidade da matéria orgânica usando os dois indicadores foram similares às obtidas in vivo em todos os experimentos. Quando as estimativas individuais, corrigidas para recuperação fecal do indicador, foram relacionadas às observações in vivo por análise de regressão, o coeficiente de regressão linear foi similar a 1 usando a MSi, mas foi menor que 1 usando a FDNi. Os valores individuais de digestibilidade da matéria orgânica estimados com os dois indicadores, contudo, foram pobremente relacionados às observações in vivo ( $\mathrm{r}^{2}$ variou de 0,60 a 0,63). Corrigindo-se para a recuperação fecal, a matéria seca residual após 144 horas de incubação in situ pode ser utilizada como indicador interno para estimar a digestibilidade média da dieta consumida por um grupo de animais, mas não é precisa para detectar pequenas diferenças na digestibilidade de alimentos impostas pelos tratamentos em um experimento.

Palavras-chave: consumo, digestibilidade, fibra em detergente neutro indigestível, matéria seca indigestível, recuperação fecal

\section{Evaluation of indigestible feed fractions as internal markers for predicting digestibility in lambs}

\footnotetext{
ABSTRACT - The use of indigestible dry matter (iDM) and indigestible neutral detergent fiber (iNDF) as internal markers to estimate digestibility in lambs was evaluated. Data and samples were obtained from six independent digestion trials with lambs housed in metabolism cages given ad libitum different forage and concentrate feedstuffs. Indigestible residues were determined after 144 hours of in situ incubation of feed and faeces samples. Recovery rate varied from 64.8 to $108.5 \%$ for iDM, and from 49.5 to $67.9 \%$ for iNDF. As the marker ratio between marker concentration in the faeces and the feed was not corrected for fecal recovery, the large part of the estimated apparent organic matter digestibility (AOMD) of the experimental means were lower than the in vivo that obtained. As the marker ratio was corrected for fecal recovery of the marker, the AOMD means estimated using both markers were similar to the in vivo data in al experiments. As individual estimates, corrected for fecal recovery of the marker, were related to in vivo observations through regression analysis, the slope of the regression for iDM was not different from 1 whereas for iNDF, the slope was lower than 1. Individual AOMD values estimates using both markers, however, were poorly related to the in vivo observations ( $\mathrm{r}^{2}$ varied from 0.60 to 0.63 ). Once corrected for fecal recovery, residual DM after 144 hours of in situ incubation is suitable for use as an internal marker to estimate mean diet digestibility consumed for a group of animals However, individual estimates were not accurate to detect small differences in digestibility imposed by different treatments within an experiment.
}

Key Words: digestibility, fecal recovery, indigestible dry matter, indigestible neutral detergent fiber, intake 


\section{Introdução}

O consumo de forragem é usualmente o fator mais limitante do desempenho de animais em pastejo, mas não pode ser medido diretamente em animais a pasto e, nestes experimentos, pode ser estimado pela medida da excreção fecal e pela estimativa da digestibilidade da forragem consumida (Penning \& Johnson, 1983). A digestibilidade da forragem pode ser estimada por ensaios in vitro, in situ ou pelo uso de indicadores internos. Os ensaios de digestibilidade in vitro e in situ não incluem os efeitos associativos de componentes da dieta na fermentação ruminal, tampouco os efeitos da taxa de passagem da digesta do rúmen na extensão de degradação da forragem (Cochran et al., 1986), deficiências que são superadas pelo uso dos indicadores internos (Lippke, 2002).

O uso de frações indigestíveis dos alimentos tem sido frequentemente proposto como indicadores internos de digestibilidade (Lippke et al., 2000; Landau et al., 2005; Silva et al., 2007). Não há, contudo, um método padrão para analisar as frações indigestíveis e o grau de recuperação fecal desses indicadores têm sido amplamente variável, o que compromete a precisão e exatidão das estimativas de digestibilidade(Penning \& Johnson, 1983; Cochran etal., 1986; Lippke et al., 1986; Krysl et al., 1988; Berchielli et al., 2000).

O objetivo neste trabalho foi avaliar o grau de recuperação fecal e o uso da matéria seca indigestível (MSi) e da fibra insolúvel em detergente neutro indigestível (FDNi) como indicadores internos de digestibilidade em ovinos.

\section{Material e Métodos}

Foram utilizados dados e amostras de seis ensaios de digestibilidade, independentes, com ovinos mantidos em gaiolas de metabolismo. As dietas experimentais em cada ensaio foram constituídas de azevém verde (Lolium multiflorum) suplementado ou não com caseinato de sódio, farinha de mandioca ou farelo de glúten de milho (ensaio 1); feno de kikuio (Pennisetum clandestinum) suplementado ou não com caseinato de sódio, farinha de mandioca ou ureia (ensaio 2); feno de kikuio de diversas idades de rebrota (ensaio 3); feno de capim-arroz (Echinochloa sp) de diversas idades de rebrota (ensaio 4); feno de capim-tifton 85 (Cynodon sp.) suplementado ou não com ureia e farinha de mandioca (ensaio 5) e feno de capim-tifton 85 suplementado ou não com caseinato de sódio, farinha de mandioca ou ureia (ensaio 6). Todos os experimentos foram conduzidos em duplo quadrado latino com ovinos Texel $\times$ Corriedale, machos castrados (20 a $35 \mathrm{~kg}$ de peso vivo, PV), e cada período experimental em cada ensaio teve duração de 15 dias, incluindo 10 dias de adaptação às dietas e 5 de coleta de dados e amostras.

Em todos os ensaios, o alimento oferecido, as sobras e as fezes foram medidos e amostrados diariamente durante os cinco dias finais de cada período. Todas as amostras foram secas em estufa com ventilação forçada a $55^{\circ} \mathrm{C}$ durante pelo menos 72 horas, trituradas em moinho com peneira de porosidade de $1 \mathrm{~mm}$ e armazenadas para posterior análise. Para a medida da digestibilidade in vivo, assim como para a incubação in situ, as amostras de alimentos e fezes foram compostas por animal e período em cada ensaio. $\mathrm{O}$ teor de matéria seca (MS) das amostras foi determinado em estufa a $110^{\circ} \mathrm{C}$ durante pelo menos 8 horas e a matéria orgânica (MO) foi determinada por queima em mufla a $550^{\circ} \mathrm{C}$ durante 3 horas.

Para determinação dos resíduos indigestíveis, aproximadamente $5 \mathrm{~g}$ de cada uma das amostras dos alimentos e das fezes foram pesados em duplicatas em sacos de poliamida $(10 \mathrm{~cm} \times 10 \mathrm{~cm}$, porosidade de $50 \mu \mathrm{m})$, que foram selados e incubados no rúmen de dois bovinos holandeses fistulados (aproximadamente $450 \mathrm{~kg}$ de PV), mantidos em pastagem de azevém com suplementação diária de 2,5 kg de farelo de glúten de milho (20\% de proteína bruta). Os sacos foram removidos do rúmen após 144 horas de incubação, lavados intensivamente em água corrente, secos em estufa a $110^{\circ} \mathrm{C}$ durante pelo menos 12 horas e pesados. A matéria seca residual foi considerada MSi. Para determinação da FDNi, os sacos contendo a MS residual foram tratados em seguida com solução detergente neutro (sem uso de sulfito de sódio ou amilase) durante 60 minutos (Mertens, 2002), lavados com água corrente e água destilada quente várias vezes até extração total do detergente residual, imersos em acetona durante 5 minutos, secos em estufa a $110^{\circ} \mathrm{C}$ durante pelo menos 12 horas e pesados. A digestibilidade aparente da matéria orgânica (DMO) foi estimada individualmente para cada animal, dieta e período pela relação entre a concentração de MSi ou FDNi no alimento e nas fezes:

$\mathrm{DMO}=1-\left(\left[\right.\right.$ alimento $_{\mathrm{MSi}}$ ou FDNi $] /\left[\right.$ fezes $\left.\left._{\mathrm{MSi} \text { ou FDNi }}\right]\right)$

O grau de recuperação fecal (RF) dos dois indicadores foi calculado com base na quantidade excretada dividida pela quantidade ingerida do indicador:

$\mathrm{RF}=\left(\right.$ fezes $(\mathrm{g} /$ dia $) \times\left[\right.$ fezes $_{\mathrm{MSi}}$ ou FDNi $\left.]\right) /($ alimento $(\mathrm{g} /$ dia) $\times$ [alimento $\left.{ }_{\text {MSi ou FDNi }}\right]$ )

O grau médio de recuperação fecal ( $\left.\mathrm{RF}_{\text {média }}\right)$ em cada ensaio foi usado para corrigir as estimativas individuais de digestibilidade no respectivo ensaio:

\footnotetext{
DMO corrigida $=1-\left(\left[\right.\right.$ alimento $\left._{\mathrm{MSi} \text { ou FDNi }}\right] /\left[\right.$ fezes $_{\mathrm{MSi}}$ ou

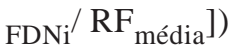


A análise de variância dos dados de recuperação fecal dos indicadores incluiu os efeitos dos ensaios e do erro experimental. Os valores médios de recuperação fecal de ambos indicadores em cada ensaio foram comparados pelo teste t para dados pareados. Esse teste também foi utilizado para comparar os valores médios de digestibilidade da matéria orgânica de cada ensaio obtidos pela coleta total das fezes ou estimados com ambos os indicadores. Os valores individuais de digestibilidade da matéria orgânica obtidos e estimados por ambos os indicadores foram comparados por regressão. Duas regressões foram utilizadas para testar a validade de cada indicador: a primeira calculou uma intercepta e gerou um $\mathrm{r}^{2}$, o qual indicou a precisão das estimativas. O desvio do coeficiente de regressão de 1 foi avaliado pelo teste $t$ bilateral. A segunda regressão foi forçada à origem (zero) e estimou o grau de desvio (bias) das estimativas. As análises foram feitas utilizando-se o PROC GLM e PROC REG do programa computacional Statistical Analysis Systems (SAS, 2004).

\section{Resultados e Discussão}

O grau médio de recuperação da MSi variou de 64,8 a $108,5 \%$ e foi maior $(\mathrm{P}<0.05)$ que o da FDNi (49,5 a 67,9\%) em todos os ensaios (Tabela 1). Quando a relação entre a concentração no alimento e nas fezes de ambos os indicadores não foi corrigida para a recuperação fecal, a maior parte das médias de digestibilidade da matéria orgânica estimadas em cada ensaio foi menor $(\mathrm{P}<0.05)$ que as obtidas pela coleta total de fezes (Tabela 2). Entretanto, quando corrigidas para a recuperação fecal, as estimativas médias de digestibilidade da matéria orgânica usando qualquer um dos indicadores foram similares às observadas em todos os ensaios de digestibilidade.

O nível de precisão das estimativas da relação entre os dados individuais de digestibilidade da matéria orgânica observados nos animais de todos os experimentos com os estimados pelos indicadores internos, indicado pelos coeficientes de determinação $\left(\mathrm{r}^{2}\right)$ das regressões $(0,60 \mathrm{e}$ 0,63 ), assim como o grau de desvio das estimativas (1 e $2 \%$ ), foi similar entre os indicadores (Figuras 1 e 2). O coeficiente de regressão linear, contudo, não foi diferente de 1 utilizando a MSi, mas foi menor que $1(\mathrm{P}<0,05)$ utilizando-se a FDNi.

Uma das características importantes de um indicador ideal é sua resistência à digestão ao longo do trato gastrointestinal (Faichney, 1975). Contudo, como também verificado em outros estudos com indicadores similares (Penning \& Johnson, 1983; Cochran et al., 1986; Lippke et al., 1986; Krysl et al., 1988; Berchielli et al., 2000), o grau de recuperação fecal da MSi e da FDNi neste estudo não foi completo e foi altamente variável entre os ensaios. Incompleta recuperação do indicador pode estar relacionada à digestão e/ou absorção parcial, à modificação físicoquímica ao longo do trato digestivo ou a deficiências dos procedimentos analíticos. Penning \& Johnson (1983) determinaram a concentração de resíduos indigestíveis em amostras moídas em peneiras com porosidade de 0,8 mm e reportaram que a incubação dessas amostras em sacos com porosidade de $43 \mu \mathrm{m}$ resultou em perdas de material pelos poros. Lippke et al. (1986) observaram que o grau de recuperação de FDNi variou entre amostras de diversos tipos de forrageiras e foi afetado, também, pelo método de determinação (incubação in vitro ou in situ). Udén (2006) verificou que a recuperação de resíduos de incubação após tratamento com detergente ácido ou neutro foi incompleta quando o material foi filtrado em cadinhos de vidro sinterizado de porosidade 2 (poros variando de 40 a $100 \mu \mathrm{m}$ ). Além disso, observou que as perdas eram maiores para amostras de fezes ou de alimentos finamente moídos (partículas menores que 0,6 mm) que para amostras de alimentos mais grosseiramente moídas (partículas entre 0,6 e $1,5 \mathrm{~mm}$ ). Neste estudo, todas as amostras foram moídas para passar em poros de $1 \mathrm{~mm}$ e a porosidade dos sacos de incubação foi em torno $50 \mu \mathrm{m}$. Assim, é provável que o baixo

Tabela 1 - Grau de recuperação dos indicadores (\%) em ovinos em diferentes experimentos

\begin{tabular}{|c|c|c|c|c|c|}
\hline \multirow[t]{2}{*}{ Experimento } & \multicolumn{4}{|c|}{ Indicador $^{1}$} & \multirow[b]{2}{*}{$\mathrm{n}$} \\
\hline & MSi & Erro-padrão ${ }^{2}$ & FDNi & Erro-padrão ${ }^{2}$ & \\
\hline 1 & $108,5 a$ & 4,8 & $60,2 b$ & 3,5 & 44 \\
\hline 2 & $76,5 \mathrm{a}$ & 1,7 & $49,9 b$ & 1,4 & 30 \\
\hline 3 & $64,8 \mathrm{a}$ & 2,7 & $49,5 b$ & 2,2 & 17 \\
\hline 4 & $99,6 a$ & 1,6 & $59,9 b$ & 1,7 & 28 \\
\hline 5 & $92,9 a$ & 2,6 & $67,9 b$ & 1,5 & 50 \\
\hline 6 & $76,7 \mathrm{a}$ & 2,1 & $50,0 \mathrm{~b}$ & 1,2 & 49 \\
\hline Média & $89,0 \mathrm{a}$ & 1,3 & $57,0 \mathrm{~b}$ & 1,9 & 218 \\
\hline
\end{tabular}

a,b: médias na mesma linha sem uma letra comum diferem significativamente $(\mathrm{P}<0,05)$.

${ }^{1} \mathrm{MSi}$, matéria seca indigestível; FDNi, fibra em detergente neutro indigestível.

2 Erro-padrão das médias. 
Tabela 2 - Digestibilidade aparente da matéria orgânica em experimentos com ovinos, obtida pela coleta total das fezes ou estimada utilizando-se a matéria seca indigestível e da fibra em detergente neutro indigestível como indicadores (MSi) ou corrigida (MSic) ou pela fibra em detergente neutro indigestível não corrigida (FDNi) ou corrigida para a recuperação fecal (FDNic)

\begin{tabular}{|c|c|c|c|c|c|c|c|c|c|}
\hline \multirow[t]{2}{*}{ Experimento } & \multirow{2}{*}{$\begin{array}{c}\text { Coleta total } \\
\text { de fezes }\end{array}$} & \multicolumn{8}{|c|}{ Indicador } \\
\hline & & MSi & Erro-padrão & MSic & Erro-padrão & FDNi & Erro-padrão & FDNic & Erro-padrão \\
\hline 1 & 0,76 & 0,77 & 0,010 & 0,75 & 0,011 & $0,57 *$ & 0,018 & 0,74 & 0,012 \\
\hline 2 & 0,59 & $0,45^{*}$ & 0,012 & 0,58 & 0,008 & $0,15^{*}$ & 0,020 & 0,58 & 0,008 \\
\hline 3 & 0,70 & $0,54 *$ & 0,011 & 0,70 & 0,011 & $0,40 *$ & 0,014 & 0,71 & 0,012 \\
\hline 4 & 0,57 & 0,56 & 0,007 & 0,57 & 0,007 & $0,26^{*}$ & 0,023 & 0,56 & 0,014 \\
\hline 5 & 0,60 & $0,54^{*}$ & 0,010 & 0,58 & 0,010 & $0,38 *$ & 0,014 & 0,58 & 0,008 \\
\hline 6 & 0,60 & $0,46^{*}$ & 0,013 & 0,58 & 0,012 & $0,18 *$ & 0,017 & 0,59 & 0,009 \\
\hline
\end{tabular}

Médias estimadas com cada indicador em cada experimento seguidas de asterisco (*) são diferentes $(\mathrm{P}<0.05)$ da média obtida pela coleta total de fezes.

MSi = matéria seca indigestível não-corrigida para recuperação fecal.

MSic = matéria seca indigestível corrigida para recuperação fecal.

$\mathrm{FDNi}$ = fibra em detergente neutro indigestível não corrigida para recuperação fecal.

FDNic = fibra em detergente neutro indigestível corrigida para recuperação fecal.

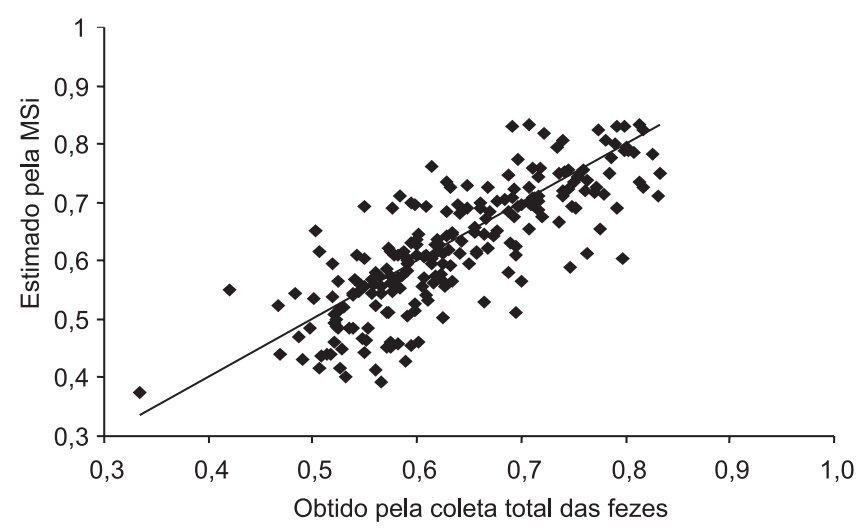

$\mathrm{Y}=0,02+0,94 \mathrm{X}$, erro-padrão do coeficiente de regressão $=0,049, \mathrm{r}^{2}=0,63$, coeficiente de regressão não diferiu de 1 . A linha no gráfico representa perfeita concordância entre os valores obtidos e estimados $(\mathrm{Y}=\mathrm{X})$. Desvio (bias) foi: $\mathrm{Y}=0,98 X . \mathrm{n}=218$.

Figura 1- Digestibilidade aparente da matéria orgânica em ovinos: relação entre os valores individuais obtidos por coleta total de fezes e os estimados pela matéria seca indigestível (MSi).

grau de recuperação, assim como as diferenças de recuperação entre os ensaios, tenha sido ocasionado pelas perdas variáveis de material fecal pelos poros dos sacos de incubação.

A viabilidade e/ou aceitabilidade do uso de indicadores internos têm sido usualmente avaliada comparando-se valores médios de digestibilidade observados e estimados em um grupo de animais de um ensaio experimental (Penning and Johnson, 1983; Cochran et al, 1986; Lippke et al., 1986; Krysl et al., 1988; Berchielli et al., 2000). Os resultados desses estudos têm indicado que, independentemente do tipo de indicador e do grau de recuperação fecal, a digestibilidade in vivo, como média de todas as observações em um experimento, pode ser estimada com

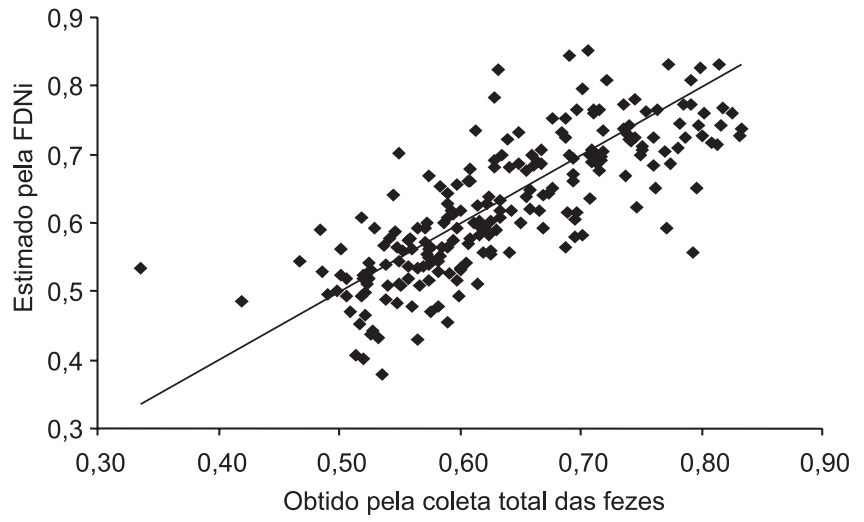

$Y=0,08+0,85 X$, erro-padrão do coeficiente de regressão $=0,048 ; r^{2}=0,60$ coeficiente de regressão foi menor que $1(\mathrm{P}<0,05)$. A linha no gráfico representa perfeita concordância entre os valores obtidos e estimados $(\mathrm{Y}=\mathrm{X})$. Desvio (bias) foi: $\mathrm{Y}=1,01 \mathrm{X}, \mathrm{n}=218$

Figura 2 - Digestibilidade aparente da matéria orgânica em ovinos: relação entre os valores individuais obtidos por coleta total de fezes e os estimados pela fibra em detergente neutro indigestível (FDNi).

alta precisão e exatidão com o uso de resíduos indigestíveis como indicadores internos, desde que o grau de recuperação do indicador seja medido e considerado no cálculo. Contudo, na maior parte dos estudos nutricionais, a unidade experimental é representada por um animal individualmente, sobre o qual os efeitos de diferentes tratamentos são medidos. A análise de regressão entre os valores de digestibilidade observados e estimados neste estudo indicam a MSi como um indicador interno mais aceitável que a FDNi para estimar a digestibilidade da matéria orgânica individual em ovinos. Os valores do $\mathrm{r}^{2}$ (60 e 63\%), de qualquer forma, indicam que pequenas diferenças de digestibilidade impostas por diferentes 
tratamentos em um experimento provavelmente não seriam detectadas com a utilização de nenhum dos indicadores testados neste estudo.

Em estudos com animais a pasto, o consumo individual de forragem pode ser estimado com base em estimativas independentes de ambos, excreção fecal de MS e digestibilidade da MS da forragem. Quando algum indicador é utilizado com o propósito de estimar a digestibilidade, usualmente são coletadas amostras fecais diretamente do reto dos animais em horários pré-determinados ao longo do dia para análise. Por esse procedimento, assume-se que a concentração fecal do indicador não varia ao longo do dia e/ou o protocolo de amostragem elimina o erro associado a uma possível variação. Neste estudo as amostras fecais foram obtidas de material representativo da excreção fecal total dos animais e, desse modo, não foi avaliado se a concentração fecal da MSi ou da FDNi varia ao longo do dia. Os resultados indicam ainda que, para melhorar o grau de exatidão das estimativas de consumo de forragem em experimentos de pastejo utilizando resíduo indigestível como indicador interno de digestibilidade, é necessário conduzir ensaios paralelos de digestibilidade para medir o grau de recuperação desse indicador. Neste caso, assume-se que a forragem ingerida e os processos digestivos nos animais do ensaio de digestibilidade são representativos daqueles que ocorrem nos animais a pasto.

\section{Conclusões}

Corrigindo-se para a recuperação fecal, a matéria seca residual após 144 horas de incubação in situ pode ser utilizada como indicador interno para estimar a digestibilidade média de uma dieta consumida por um grupo de animais, mas é pouco precisa para detectar pequenas diferenças na digestibilidade de alimentos impostas pelos tratamentos em um experimento.

\section{Literatura Citada}

BERCHIELLI, T.T.; ANDRADE, P.; FURLAN C.L. Avaliação de indicadores internos em ensaios de digestibilidade. Revista Brasileira de Zootecnia, v.29, p.830-833, 2000.

COCHRAN, R.C.; ADAMS, D.C.; WALLACE J.D. et al. Predicting digestibility of different diets with internal markers: evaluation of four potential markers. Journal of Animal Science, v.63, p.1476-1483, 1986.

FAICHNEY, G.J. The use of markers to partition digestion within the gastro-intestinal tract of ruminants. In: McDONALD I.W.; WARNER, A.C.I. (Eds.) Digestion and metabolism in the ruminant. Armidale: The University of New England Publishing Unit, 1975. p.277-291.

KRYSL, L.J.; GALYEAN, M.L.; ESTELL R.E. et al. Estimating digestibility and faecal output in lambs using internal and external markers. Journal of Agricultural Science, v.111, p.19-25, 1988.

LANDAU, S.; KABABYA, D.; SILANIKOVE, N. et al. The ratio between dietary rumen degradable organic matter and crude protein may affect milk yield and composition in dairy sheep. Small Ruminant Research, v.58, p.115-122, 2005.

LIPPKE H. Estimation of forage intake by ruminants on pasture. Crop Science, p.42, p.869-72, 2002.

LIPPKE, H.; FORBES, T.D.A.; ELLIS, W.C. Effect of supplements on growth and forage intake by stocker steers grazing wheat pasture. Journal of Animal Science, v.78, p.1625-1635, 2000.

LIPPKE, H.; ELLIS, W.C.; JACOBS B.F. Recovery of indigestible fiber from feces of sheep and cattle on forage diets. Journal of Dairy Science, v.69, p.403-412, 1986.

MERTENS, D.R. Gravimetric determination of amylase-treated neutral detergent fibre in feeds with refluxing beakers or crucibles: a collaborative study. Journal of AOAC, v.85, p.1217-1240, 2002.

PENNING, P.D.; JOHNSON, R.H. The use of internal markers to estimate herbage digestibility and intake. Journal of Agricultural Science, v.100, p.127-131, 1983.

SILVA, E.A.; BERCHIELLI, T.T.; REIS, R.A. et al Teores de proteína bruta para bovinos alimentados com feno de tifton 85: consumo e digestibilidades total e parcial. Revista Brasileira de Zootecnia, v.36, p.237-245, 2007.

STATISTICAL ANALYSIS SYSTEM - SAS. SAS/STAT ${ }^{\circledR}$ User's guide. Version 9.1. Cary: SAS Institute, 2004. 5136p.

UDÉN, P. Recovery of insoluble fibre fractions by filtration and centrifugation. Animal Feed Science and Technology, v.129, p.316-328, 2006. 